\title{
A Study of Uncertainty Contribution to Cryptocurrency Investment Dynamics
}

\author{
Polyakova Aleksandra ${ }^{1}$, Zavyalov Dmitry ${ }^{1}$, Kolmakov Vladimir ${ }^{1}{ }^{*}$ \\ 1Plekhanov Russian University of Economics; 36, Stremyanny lane, Moscow, 117997, Russian Federation
}

\begin{abstract}
We investigate the interrelation between the economic policy uncertainty index and composite cryptocurrency index to contribute to the contemporary discussion and verify the available results of other researchers. Our research objective is to verify the hypothesis about the positive correlation between uncertainty and cryptocurrency price with regard to the previously published results. We employ a trailing correlation and linear regression of data at different lags to find evidence of co-movement and, if found, to determine the nature of cryptocurrency investment-a safe haven or abnormal return-seeking venture. We employ the most recent data (ending May 2021) and come to a conclusion that requires revisiting the known findings, as our results at earlier levels in the time series follow the mainstream, while the most recent data prove them wrong. The major conclusion made upon completion of the analysis is that there is no reliable correlation between economic policy uncertainty and cryptocurrency exchange rates, which could have practical usability.
\end{abstract}

Keywords: Cryptocurrency; Risk prediction; Trailing correlation; Uncertainty index

\section{Introduction}

The United Nations Organization denoted cryptocurrency as the new frontier in finance. According to the United Nations, cryptocurrency and blockchain technology can create breakthrough business models that eliminate or augment routines and significantly contribute to performance and efficiency, shaping the global "digital future" (Bencsik, 2020). As is well known, its practical implementations are spreading from decentralized finance products to corporate social responsibility management issues (see, e.g., Kulkova, 2020, or Berawi, 2020b), as cryptocurrency markets have developed significantly and provided many reasons to investigate their development drivers and factors.

One opinion holds that cryptocurrencies and related assets can provide investors with a powerful instrument of risk mitigation in periods of volatility and unpredictability, which has increased significantly under COVID-19 circumstances, causing many governments to take necessary actions and collaborate to enable robust global recovery (Berawi 2020a). Alternatively, one can assume that cryptocurrencies are not "safe havens" but are profitseeking ventures that aim for maximum yields when major investors rebalance their portfolios in favor of conservative assets.

To formalize, we assume that an interdependence exists between cryptocurrency returns and economic uncertainty. Thus, we posit the following research hypothesis: Increases in economic uncertainty contribute positively to cryptocurrency returns;

${ }^{*}$ Corresponding author's email: Kolmakov.VV@rea.ru, Tel.: +7-925-3528503 doi: 10.14716/ijtech.v12i7.5348 
alternatively, decreases in uncertainty undermine investors' faith in cryptocurrency in favor of traditional segments of the market.

This hypothesis is not a brand-new development in contemporary science. Several papers have employed the same methodological approach to test cryptocurrency responses to uncertainty changes. Namely, Wu et al. (2021) applied Twitter-based economic policy uncertainty measures to test the Granger causality presence in uncertainty influence on cryptocurrencies and found that the influence was positive. Haq et al. (2021) concluded that economic policy uncertainty implies different patterns of correlation with cryptocurrency market development, depending on national-specific features, such as regulation or financial market alternatives for risk mitigation.

Many researchers agree that the cryptocurrency market can respond to uncertainty change differently depending on the direction of the change. Colon et al. (2021) approached the issue from the reverse perspective; they proved that "the cryptocurrency market can serve as a strong hedge against geopolitical risks in most cases, but it could be considered a weak hedge and safe haven against economic policy uncertainty during a bull market." In other words, they considered cryptocurrency to be a factor of instability. The variability of correlations between cryptocurrency and uncertainty was also demonstrated by Qian et al. (2021), who explored the economic policy uncertainty index as an external factor for possibly explaining correlations between the cryptocurrency index (CRIX) and the world stock market portfolio. Their findings demonstrate that "the correlation is influenced by the uncertainty stance of the economy and behaves differently in low-, medium-, and highuncertainty periods."

Concordant to the former and the latter, Koumba et al. (2020) found that "economic policy uncertainty affects exchange rates on cryptocurrency assets in times of financial turbulence characterized by low confidence in the financial stock markets, and tranquil periods where the financial stock markets behave smoothly."

Developing on that, Yen and Cheng (2021) applied a country-specific approach to address economic policy uncertainty with regard to cryptocurrency volatility. They found that uncertainty can be a valid predictor of cryptocurrency volatility in China but not in the United States or Japan. They also showed in Cheng and Yen (2020) that national economic policy uncertainty indices are improper predictors of cryptocurrency returns, except in China. Notable is the evidence provided by Phan et al. (2018), whose findings also proved that economic policy uncertainty provides different contributions to financial market dynamics across different countries.

Given all the facts above, we aim to contribute to the theory by introducing an alternative analytical approach and means of results verification.

\section{Methodology}

\subsection{Methods and Approaches}

To formalize the phenomenon of uncertainty, we employed the widely accepted methodology of economic policy uncertainty indices proposed by Baker et al. (2016). The methodology analyzes recognized business-media discourse to find the frequency and sentiment of specific words used (words relative to crises, instability, market fluctuations, risks, and threats). The formal indicator, then, is either the absolute or somehow weighted number of word-use cases. We suggest using the global economic policy uncertainty index's (GEPUI) monthly values as the potential argument that presumably influences cryptocurrency investment. From an economic point of view, this approach seems reasonable, as the main risk of uncertainty is instability and the growth of the span of fluctuations, which complicates the decision-making process, the options of which can be 
inertial-conservative or proactive. The described approach to measuring uncertainty is well known and widely used, for example, in Fang et al. (2020) or in Cheng and Yen (2020).

To address the hypothesis, we analyzed the trailing change of correlation coefficients between the two time series: GEPU index and the USD values of the S\&P Cryptocurrency Broad Digital Market Index (CBDMI) (Standard and Poor's, 2017). We tested trailing changes of correlations over time intervals of different durations: 12 months, 18 months, 2 years, and 3 years. For shorter lags, the short-term fluctuations are more explicit, and the influence of significant bias is excluded. As the lag length increases, the short-term effects will level out, and the autoregressive effect will increase, signaling the presence of a path dependence.

The trailing correlation algorithm analyzes the time series on retrospectively limited by the lag length. It is formalized in the following manner:

$$
r_{t}=\frac{\sum_{t=0}^{t-k}\left(x_{t}-\bar{x}\right)\left(y_{t}-\bar{y}\right)}{\sqrt{\sum_{t=0}^{t-k}\left(x_{t}-\bar{x}\right)^{2} \sum_{t=0}^{t-k}\left(y_{t}-\bar{y}\right)^{2}}}
$$

retrospective length index; $k=\operatorname{lag}-1$; lag is the lag length in months; and lag $\in[12 ; 18 ; 24$; 36].

The outcome interpretation was as follows. The correlation coefficient attributable to May 2021 was derived from the May 2021 value and preceding 11 values (or 17, 23, 35 values, depending on the lag length). The change in correlation between consecutive periods indicated increases or decreases in risk-taking among the investors in response to changes in uncertainty. Lags were introduced to test the rigidity of the outcomes. A negative value of the correlation coefficient indicated a decrease in cryptocurrency investment in response to increased uncertainty, and vice versa.

Absolute values of the linear regression of the CBDMI by the GEPUI coefficient were also analyzed; the bigger they are, the more incentives investors see to expand to cryptocurrency assets. We expected that earlier periods' absolute values would be less than those of later periods, which would confirm the hypothesis.

Our choice of a linear regression model was based on the proposition that the regression coefficients derived would be explanatory in terms of "executive" interpretation and instrumental in determining the presence of factor-function interrelation prior to more sophisticated models' composition. The impact-response interpretation of linear regression models is the most distinct and clear in economic analysis and decision-making, even though it disregards several statistically important effects that could be discovered using autoregressive conditional heteroskedasticity (ARCH) models. We realize that financial time series quite frequently have unit root and ARCH effects present. We did not need to reproduce generally known conclusions about volatility clustering, yet our concern was to approach the mechanism of uncertainty transmission and discover basic interrelations that were biased depending on the length of the retrospective. Further introduction of ARCH effects to our models would be possible if our hypothesis proved to be true and, what is more important, if a reliable substitute for the monthly uncertainty index would be found to benefit from a daily time series analysis.

Another point to mention is the reason for using the zero-intercept model of linear regression. We tested both original and log-transformed data and found that the intercept was up to 30 times bigger than the regression coefficient, which meant a significant contribution from side effects that we had no intention to study. Thus, the zero-intercept model allowed us to disregard side effects and search into trailing correlation issues only.

Our approach was first implemented by Polyakova (2021) and further developed by Hassan et al. (2021), who analyzed the time-varying correlations between gold quotes and 
cryptocurrency uncertainty indices to show that gold "has a stable and reliable safe-haven property against cryptocurrency uncertainty." Even though the underlying concept in the quoted article is quite different from ours, following their findings and controlling the quality of outcomes, we suggest applying our algorithm to different dependent variables, such as the S\&P500 index, Bitcoin quotes, and gold spot prices.

\subsection{Data Sources and Transformations}

For hypothesis verification, the following data were used:

- closing quotes for the CBDMI on the last trading day of a month, starting February 2017 (the earliest value available) through May 2021 (the latest value available for the GEPUI);

- absolute GEPUI monthly values in the current (unweighted) representation retrieved from the Economic Policy Uncertainty website;

- Bitcoin closing quotes on the last trading day of a month, retrieved from Yahoo;

- S\&P500 index closing values on the last trading day of a month, retrieved from Yahoo; and

- Gold spot prices on the last trading day of a month, retrieved from Yahoo.

Given the data availability constraints, all the time series had 52 valid observations.

The data were seasonally adjusted using the X11/Y2k Monthly Seasonal Adjustment (Census Method II) algorithm in Statistica 6.0 by Statsoft Inc. To control the quality of the estimates, several other transformations were tested, that is, first-order differences, relative changes of levels, and unmodified series.

\section{Results and Discussion}

\subsection{Uncertainty and Cryptocurrency Market Patterns of Development}

The GEPUI and CBDMI seem uncorrelated if looked at from the point of view of the monthly relative changes. The whole series correlation ratio equals minus 0.069 in levels and minus 0.19 in growth rates. Still, the linear regression coefficient of CBDMI dependence on GEPUI change is 3.85 in levels, which is quite large, though statistically insignificant, due to low R-squared and failed t-tests. Proper evidence comes with the plot of the monthly growth rates of the two series (see Figure 1).

Still, the cryptocurrency index is twice as volatile. Its monthly yields have a standard deviation of $32.3 \%$, while uncertainty remains within a $17.1 \%$ boundary. According to the mean value, the uncertainty index fluctuates around the zero-slope trend, while cryptocurrencies follow the general upward trend. Details are available in Table 1.

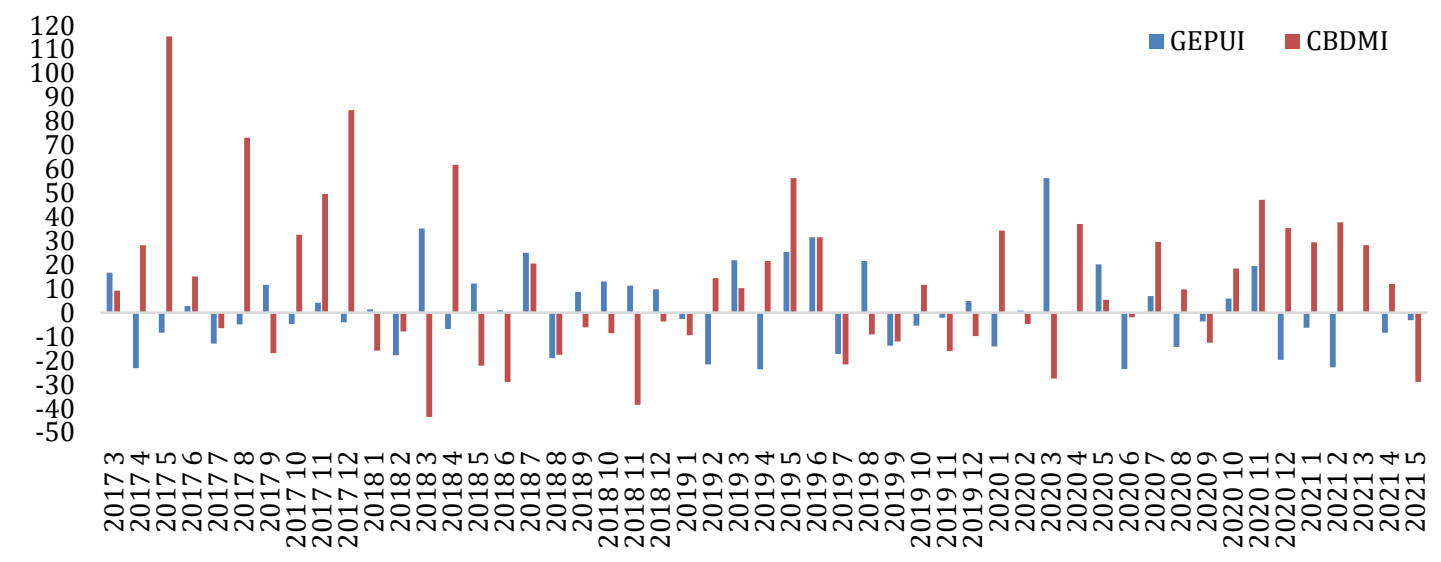

Figure 1 Monthly growth rates of GEPUI and CBDMI, \% 
Table 1 Growth rate distribution of factors in scope, monthly percentage yields

\begin{tabular}{lccccc}
\hline \multicolumn{1}{c}{ Metrics } & GEPUI & CBDMI & Bitcoin & S\&P500 & Gold \\
\hline Minimum & -23.7 & -43.6 & -36.4 & -12.5 & -5.0 \\
Maximum & 56.2 & 115.4 & 69.6 & 12.7 & 6.6 \\
Spread & 79.9 & 158.9 & 106.0 & 25.2 & 11.6 \\
Standard deviation & 17.1 & 32.3 & 26.7 & 4.63 & 2.91 \\
Mean & -0.12 & 7.2 & 7.07 & 1.18 & 0.79 \\
\hline
\end{tabular}

The first conclusion we can make using the data above is that cryptocurrency investment, according to the CBDMI dynamics, does not match the pattern of a "safe haven," which is traditionally associated with gold - the least volatile asset in our sample. The latter means that cryptocurrency assets are highly unlikely to be the resort for keeping funds in during turbulent periods. Instead, the well-known volatility of cryptocurrencies stimulates rent-seeking investors to act in the opposite manner to conservative ones; their response to uncertainty growth is the extension of long positions in cryptocurrency, that is, an opportunity to receive an abnormal return. Still, we cannot fully agree with Wu et al. (2019), who concluded that "neither gold nor Bitcoin can serve as a strong hedge or safe haven for economic policy uncertainty at the average condition." The methodology we have applied allows for tracing changes in the indices' patterns that happen in specific time intervals.

\subsection{Hypothesis Verification}

Our results from the trailing correlation analysis at different lags allow us to conclude that the two indices show different extents and directions of mutual relatedness at different periods that are highly likely to match the cryptocurrency market situation that spans from pessimism to optimism. As well, it can also be evidence of ARCH effects present in cryptocurrency index time series, which are amplified by uncertainty boosts or periods of decreases.

Still, we can obviously see that the influence of uncertainty on cryptocurrency quotes mainly weakens as lag increases. Figure 2 illustrates that, in appropriate periods, a longerlag trailing correlation ratio is lower in absolute terms than the 12-month trailing, except for some specific periods of 2020, when there was a boost of cryptocurrency optimism together with decreases of uncertainty.

The latter can be interpreted in the following manner. We see indirect proof of a volatility clustering phenomenon that presumes the need for further investigation of ARCH effects within the time series as well as its spillover to uncertainty (in terms of vector autoregressive models) and its response-slowdown or amplification-to uncertainty change. 


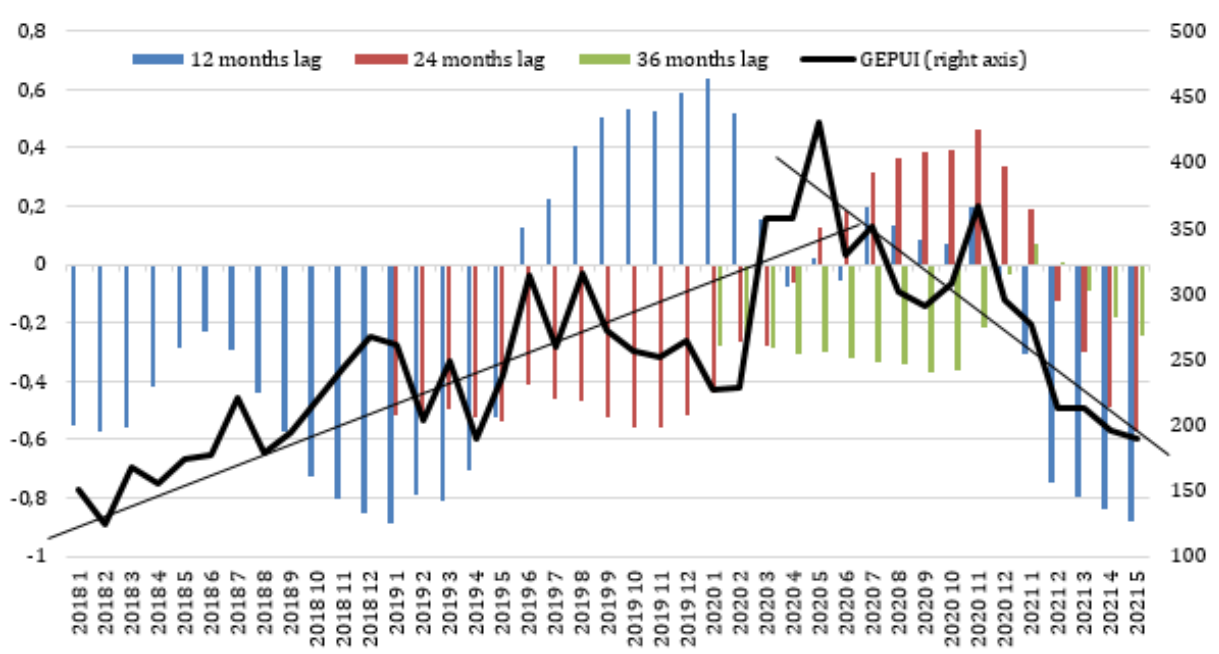

Figure 2 Correlation coefficients of GEPUI and CBDMI levels at lags of 12, 24, and 36 months

Different distributions of correlations over time require description and, presumably, explanation.

Longer-lag correlations tend to be in a counterphase. Such a finding contradicts the main issue we are trying to address-cryptocurrency quotes were meant to follow changes of uncertainty in the same direction. Instead, they behave as traditional financial assets.

An outlier situation, starting June 2019 and ending March 2020, when uncertainty was heading down, was characterized by short-lag correlations' positive values. Combined together, the factors illustrate that, until Summer 2020, the cryptocurrency market was almost indifferent to the direction of uncertainty, thus making it a low-quality factor in predicting Bitcoin or other asset fluctuations in terms of hedging the risk.

Our hypothesis then appears to be rejected; there is no reliable interrelation between uncertainty and cryptocurrency quotes. Earlier studies have provided evidence of the same nature: "We find that risk spillover effect from economic policy uncertainty to Bitcoin is negligible in most conditions" (Wang et al., 2019). The value of our contribution increases based on this contrast with other research, such as Demir et al. (2018), who indicate that "economic policy uncertainty has a predictive power on Bitcoin returns that are negatively associated with the economic policy uncertainty; thus, Bitcoin can serve as a hedging tool against uncertainty." However, an argument in favor of our hypothesis can be drawn from linear regression analysis results. We applied growth rate normalization to cryptocurrency returns and regressed them using the GEPUI.

First, we see different patterns of monthly percentage yield responses to uncertainty change in earlier and more recent periods, which means that "accumulated" uncertainty has prolonged effects, and over time, markets become more tolerant to uncertainty. Uncertainty becomes less influential on cryptocurrencies and traditional financial assets, unless the new distress factor is introduced (as COVID-19 pandemic).

Second, we see that traditional markets are significantly more tolerant to uncertainty change in short-term retrospectives. Regression coefficients are moderately volatile at around zero. S\&P500 monthly percentage yields within the analyzed retrospective have regression coefficient spans between $(-0.004)$ and 0.012 ; the same of gold's-between $(-0.005)$ and 0.009 , while cryptocurrencies' index yield varies within $(-0.049)$ and 0.187 , but still the values of regression coefficients are exceptionally low and statistically unreliable. During the flat-trend period, the patterns match (as seen in Figure 3), but as factors of distress appear, cryptocurrencies' reaction to uncertainty grows, while 
traditional assets demonstrate moderate growth in the statistical response rate to uncertainty.

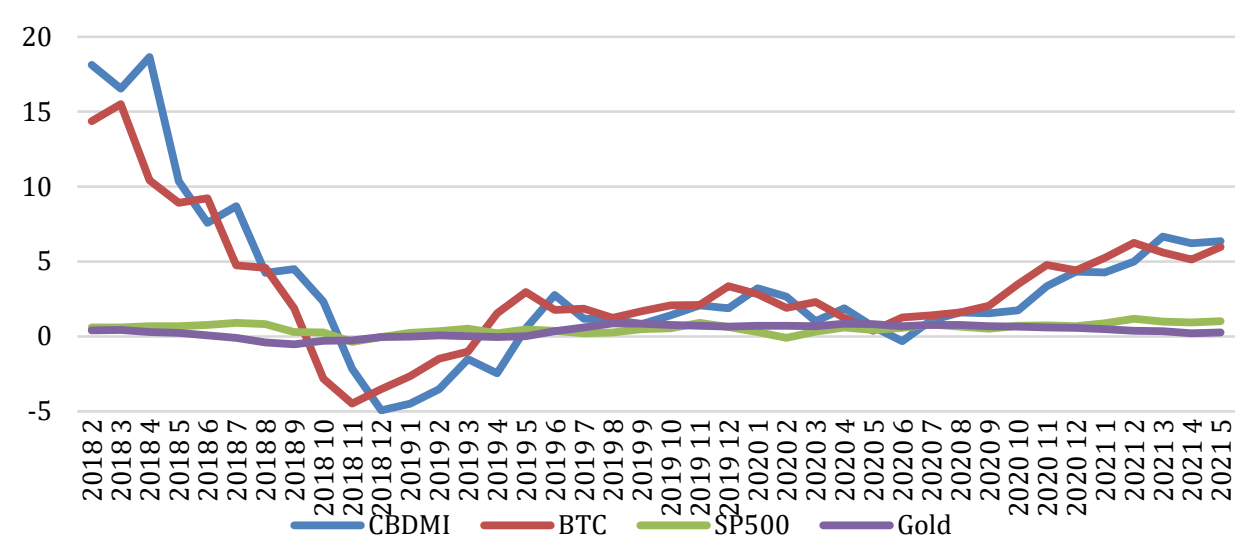

Figure 3 Linear regression coefficients at GEPUI at 12-month lag, 100x

Our findings are in line with Colon et al. (2021), who found that "the cryptocurrency market reacts to uncertainty differently, depending on the type of uncertainty." However, we disagree that uncertainty is an essential determinant of cryptocurrency returns.

Alternatively, Antonakakis et al. (2019) found that periods of high market uncertainty correspond to strong connectedness between cryptocurrencies. We can agree with that, as Bitcoin and CBDMI (which, of course, includes a significant share of Bitcoin) have very similar patterns, and Bitcoin is obviously driving the change, while other currencies in the index follow it.

\section{Conclusions}

Despite our expectations, the analysis we made makes us reject our hypothesis. Fluctuations of economic uncertainty contribute to cryptocurrency returns not in a single manner and cannot be used to explain cryptoasset growth or downgrades. With respect to the quoted research papers we can give at least one plausible explanation of the differences we see: the time series we employed were more actual. Up to some periods, our findings did fit into the mainstream, but the most recent fluctuations in the cryptocurrency market do not allow us to conclude that there is a distinct uniform pattern of correlation that can be further employed for practical purposes.

\section{References}

Antonakakis, N., Chatziantoniou, I., Gabauer, D., 2019. Cryptocurrency Market Contagion: Market Uncertainty, Market Complexity, and Dynamic Portfolios. Journal of International Financial Markets, Institutions and Money, Volume 61, pp. 37-51

Baker, S.R., Bloom, N., Davis, S.J., 2016. Measuring Economic Policy Uncertainty. Quarterly Journal of Economics, Volume 131(4), pp. 1593-1636

Bencsik, A., 2020. Challenges of Management in the Digital Economy. International Journal of Technology, Volume 11(6), pp. 1275-1285

Berawi, M.A., 2020a. Empowering Healthcare, Economic, and Social Resilience during Global Pandemic Covid-19. International Journal of Technology, Volume 11(3), pp. 436439

Berawi, M.A., 2020b. Managing Nature 5.0: The Role of Digital Technologies in the Circular Economy. International Journal of Technology, Volume 11(4), pp. 652-655 
Cheng, H.-P., Yen, K.-C., 2020. The Relationship between the Economic Policy Uncertainty and the Cryptocurrency Market. Finance Research Letters, Volume 35, https://doi.org/10.1016/j.frl.2019.101308

Colon, F., Kim, C., Kim, H., Kim, W., 2021. The Effect of Political and Economic Uncertainty on the Cryptocurrency Market. Finance Research letters, Volume 39, https://doi.org/10.1016/j.frl.2020.101621

Demir, E., Gozgor, G., Lau, C.K.M., Vigne, S.A., 2018. Does Economic Policy Uncertainty Predict the Bitcoin Returns? An Empirical Investigation. Finance Research Letters, Volume 26, pp. 145-149

Fang, T., Su, Z., Yin, L., 2020. Economic Fundamentals or Investor Perceptions? The Role of Uncertainty in Predicting Long-Term Cryptocurrency Volatility. International Review of Financial Analysis, Volume 71, https://doi.org/10.1016/j.irfa.2020.101566

Haq, I.U., Maneengam, A., Chupradit, S., Suksatan, W., Huo, C., 2021. Economic Policy Uncertainty and Cryptocurrency Market as a Risk Management Avenue: A Systematic Review. Risks, Volume 9(9), pp. 1-24

Hassan, M.K., Hasan, M.B., Rashid, M.M., 2021. Using Precious Metals to Hedge Cryptocurrency Policy and Price Uncertainty. Economics Letters, Volume 206, https://doi.org/10.1016/j.econlet.2021.109977

Koumba, U., Mudzingiri, C., Mba, J., 2020. Does Uncertainty Predict Cryptocurrency Returns? A Copula-Based Approach. Macroeconomics and Finance in Emerging Market Economies, Volume 13(1), pp. 67-88

Kulkova, V.Y., 2020. The Implementation of Internal Corporate Social Responsibility of Entrepreneurial Structures in Innovative HR Management. In: Bogoviz A. (eds) Complex Systems: Innovation and Sustainability in the Digital Age. Studies in Systems, Decision and Control, vol 282. Springer, Cham. pp. 19-24

Phan, D.H.B., Sharma, S.S., Tran, V.T., 2018. Can Economic Policy Uncertainty Predict Stock Returns? Global Evidence. Journal of International Financial Markets, Institutions and Money, Volume 55, pp. 134-150

Polyakova, A.G., 2021. Change of the Population Financial Behavior under the Coronavirus Pandemic and Economic Crisis. Karelian Scientific Journal, Volume 10(2), pp. 25-29

Qian, L., Jiang, Y., Long, H., Song, R., 2021. The Roles of Economic Policy Uncertainty and the COVID-19 Pandemic in the Correlation Between Cryptocurrency and Stock Markets. Singapore Economic Review, pp. 1-30

Standard \& Poor's, 2017. S\&P Cryptocurrency Broad Digital Market Index. Available Online at https://www.spglobal.com/spdji/en/indices/digital-assets/sp-cryptocurrencybroad-digital-market-index/\#overview, Accessed on December 08, 2021

Wang, G.-J., Xie, C., Wen, D., Zhao, L., 2019. When Bitcoin Meets Economic Policy Uncertainty (EPU): Measuring Risk Spillover Effect from EPU to Bitcoin. Finance Research Letters, Volume 31, pp. 489-497

Wu, S., Tong, M., Yang, Z., Derbali, A., 2019. Does Gold or Bitcoin Hedge Economic Policy Uncertainty? Finance Research Letters, Volume 31, pp. 171-178

Wu, W., Tiwari, A.K., Gozgor, G., Leping, H., 2021. Does Economic Policy Uncertainty Affect Cryptocurrency Markets? Evidence from Twitter-Based Uncertainty Measures. Research in International Business and Finance, Volume 58, https://doi.org/10.1016/j.ribaf.2021.101478

Yen, K.-C., Cheng, H.-P., 2021. Economic Policy Uncertainty and cryptocurrency Volatility. Finance Research letters, Volume 38, https://doi.org/10.1016/j.frl.2020.101428 